\title{
HOXD3 Mediates the Switchover of Cadherin 4 to $\beta 3$ Integrin Gene Expression in Human Erythroleukemia HEL Cells
}

\author{
Yasushi Taniguchi ${ }^{1}$, Tetsuya Moriuchi ${ }^{2}$, Hidetoshi Inoko ${ }^{1}$ and Minoru Kimura ${ }^{1}$ \\ ' Division of Basic Molecular Science and Molecular Medicine, School of Medicine, Tokai University, Bohseidai, Isehara, Kanagawa 259- \\ 1193, Japan and ${ }^{2}$ Division of Cancer-related Genes, Institute for Genetic Medicine, Hokkaido University, Sapporo, Japan \\ (Received $16 \mathrm{May}$ 2003; and Accepted 29 May 2003)
}

\begin{abstract}
We previously reported that overexpression of the HOXD3 homeobox gene in human erythroleukemia HEL cells gave rise to changes in cell adhesiveness. In the present study, we examined the consequences of over- or underexpression of HOXD3 in HEL cells for colony morphology, the ability to adhere to fibronectin, and expression of cell adhesion molecules in order to further confirm the effects attributed to varied levels of HOXD3 expression. Cells overexpressing HOXD3, which formed compact colonies composed of condensed cells, had a greater affinity for fibronectin and expressed higher levels of $\beta 3$ integrin mRNA than the control cells transfected with an empty vector. Cells which did not express any HOXD3 mRNA failed to form colonies, had a low affinity for fibronectin, and expressed low levels of $\beta 3$ integrin mRNA. Instead, the cells underexpressing HOXD3 exhibited higher levels of cadherin $4 \mathrm{mRNA}$ than the control and cells overexpressing HOXD3. These results demonstrate that the ability to form colonies and attach to fibronectin in HEL cells was dependent on levels of HOXD3 expression, and that HOXD3 served as a switch from cadherin 4 to $\beta 3$ integrin gene expression.
\end{abstract}

HOX genes encode DNA-binding proteins which function as transcriptional regulators. In mammals such as human and mouse, 39 HOX genes are organized in four separate gene clusters called HOX A, B, $\mathrm{C}$, and $\mathrm{D}$. These clusters are presumed to have arisen by repeated duplication of an ancestral complex (10). Individual members of HOX genes in the four clusters are classified into 13 paralogous

Correspondence to: Dr. Yasushi Taniguchi

Division of Basic Molecular Science and Molecular Medicine, School of Medicine, Tokat University, Bohsei-

dai, Isehara, Kanagava 259-1193, Japan

Tel: +81-463-93-1121 Fax: +81-463-96-2892

E-mail: ytanigu@is.icc.u-tokai.ac.jp

Abbreviations: HEL, human erythroleukemia; RTPCR, reverse transcriptase-mediated polymerase chain reaction; dCTP, deoxycytidine 5'-triphosphate; G3PDH, glyceraldehyde 3-phosphate dehydrogenase; BLAST, basic local alignment search tool; DDBJ, DNA data bank of Japan; PBS, phosphate-buffered saline groups, according to the similarity of their nucleotide sequences and the position of the genes within the clusters (19). As for members which belong to the different paralogous groups, there is a "colinear" relationship between an anteroposterior pattern of embryonic expression domains, a so-called HOXcode, and ordering of the genes within each complex (15). A number of mutational analyses show that qualitative and quantitative differences of the HOX-code in embryonic segments along the anteroposterior axis play a pivotal role in determining the regional specificity of cell fate $(5,12,13,16)$.

The HOXD3 (Hoxd-3) gene, a third paralogous member of the HOX D family, has been structurally analyzed in human $(22,23)$, mouse $(3,21)$, and chicken (20). Targeted disruption of the mouse Hoxd-3 gene exhibits a homeotic transformation of the craniocervical joint, as a result of aberrant mesenchymal condensation during skeletal development (4). On the other hand, in cell biological studies, 
overexpression of the human HOXD3 gene has been reported to give rise to changes in cell adhesiveness and elevation of $\beta 3$ integrin gene expression in erythroleukemia cells (24), umbilical vein endothelial cells (2), and lung cancer cells (9). However, it is unclear how such cellular events caused by elevated levels of HOXD3 gene expression are implicated in the phenotype of mice deficient in HOXD3 expression.

In the present study, we found that a deficiency of HOXD3 gene expression prevented HEL cells from condensation. We simultaneously observed elevated expression of cadherin $4 \mathrm{mRNA}$ and decreased expression of $\beta 3$ integrin mRNA. The possible function of HOXD3 at a cellular level is discussed.

\section{MATERIALS AND METHODS}

Cells and cell culture. Human erythroblastic leukemia HEL cells were provided by the Japanese Cancer Research Resources Bank (JCRB; Tokyo, Japan). The cells were maintained in RPMI 1640 (Sigma, St. Louis, Mo, USA) medium supplemented with $10 \%$ heat-inactivated fetal bovine serum (Cytosystems, New South Wales, Australia) in a humidified $5 \% \mathrm{CO}_{2}$ incubator. For most cultures, negatively charged tissue-culture flasks (Iwaki Glass, Chiba, Japan) were used. For cell adhesion assays, fibronectin-coated dishes (Iwaki Glass, Chiba, Japan) were used.

Construction of expression vectors. The expression vectors were constructed according to the method described previously (24). In brief, the HOXD3 genomic DNA, including the full coding region and intron, was inserted into a mammalian expression vector, pMAMneo (Clontech, Palo Alto, CA, USA), in the sense orientation (pMAMneo-HOXD3 sense) and in the antisense orientation (pMAMneoHOXD3 antisense).

Transfections and cell cloning. Transfections were performed using a Lipofectin Kit (Boehringer, Mannheim, Germany) according to the method described earlier (24). Stable transfectants were selected in the presence of $600 \mu \mathrm{g} / \mathrm{ml}$ G418 (GIBCO, Gland Island, NY, USA). Cells were cloned by limiting dilution with 96-well plastic plates (Costar, Cambridge, MA, USA). Several control transfectants (neo), into which the unmanipulated vector (pMAMneo) was introduced, were isolated. Of clones transfected with the pMAMneo-HOXD3 sense vector, three clones overexpressing HOXD3 (S1, S2, and S3) were se- lected. Of clones transfected with the pMAMneoHOXD3 antisense vector, two clones (AS1 and AS3) were completely lacking in HOXD3 expression, while a clone (AS2) expressed a lower level of HOXD3 than the control transfectants.

$R N A$ isolation and RT-PCR. To isolate total RNA from cultured cells, the cells were lysed in a buffer containing $10 \mathrm{mM}$ Tris- $\mathrm{HCl}(\mathrm{pH} \quad 8.5), 1.5 \mathrm{mM}$ $\mathrm{MgCl}_{2}, 140 \mathrm{mM} \mathrm{NaCl}, 0.5 \%$ Nonidet-P40, $1 \mathrm{mM}$ dithiothreitol, and 1,000 units/ml placental RNase inhibitor (Toyobo, Osaka, Japan), and the cytoplasmic extracts were treated according to the method described by Sambrook et al. (17). RT-PCR was carried out according to the manufacturer's protocol in an RNA PCR system (Perkin-Elmer Cetus, Nolwalk, CT, USA). A total of $1 \mu \mathrm{g}$ of cytoplasmic RNA was reverse-transcribed using random hexanucleotides as primers. The cDNA was amplified using $10 \mathrm{nM}$ forward and reverse primers. PCRs were run in the Iwaki thermal sequencer TSR-300 (Iwaki Glass, Chiba, Japan) at $94^{\circ} \mathrm{C}$ for $1 \mathrm{~min}, 60^{\circ} \mathrm{C}$ or 65 ${ }^{\circ} \mathrm{C}$ for $1 \mathrm{~min}$, and $72{ }^{\circ} \mathrm{C}$ for $1 \mathrm{~min}$ for the desired number of cycles for the various cDNAs, followed by $72^{\circ} \mathrm{C}$ for $10 \mathrm{~min}$. The PCR products were separated by $2 \%$ agarose or $7.5 \%$ polyacrylamide gel electrophoresis. For a quantitative analysis, PCR was performed within the range of the number of cycles which had a linear relationship with the quantity of the products (24). Primer pairs used for PCR amplification were as follows: HOXD3 forward primer, 5'-TGGTCTGAACTCAGAGCAGCAG CCA-3'; HOXD3 reverse primer, 5'-ACTTCATGCG CCGGTTCTGGAACCA-3'; cadherin forward primer, 5'-GAAGAAGGTGGAGGAGAAGAAGACC-3'; cadherin reverse primer, 5'-ACCATACATGTCCGCC AGTTTCTTG-3'; cadherin 4 forward primer, 5'-AA GCCATGGGGCACGTGCCAAGCAA-3'; cadherin 4 reverse primer, 5'-TCGTTGAGGTAATCGTAGTC TTGGT-3'; $\beta$-actin forward primer; $5^{\prime}$-GGAGA AAATCTGGCACCACACCTTC-3'; $\beta$-actin reverse primer, 5'-TCTCCTTAATGTCACGCACGATTTC3'; G3PDH forward primer, 5'-ACCACAGTCCATG CCATCAC-3'; G3PDH reverse primer, 5'-TCCAC CACCCTGTTGCTGTA-3'.

Southern and Northern blot hybridization. For Southern analysis, RT-PCR products were separated by $2 \%$ agarose gel electrophoresis, and were transferred onto nylon filters. For Northern analysis, a total of $20 \mu \mathrm{g}$ cytoplasmic RNA was electrophoresed on $1 \%$ agarose-formaldehyde gels, and the bands were transferred onto nylon filters. In both cases, 
the filters were hybridized and washed as described previously (25). DNA probes for HOXD3, $\beta 1$ and $\beta 3$ integrin, and $\beta$-actin were mentioned previously (24). The mouse R-cadherin cDNA probe (14) was a gift from Dr. M. Takeichi (Kyoto University, Kyoto, Japan). To detect G3PDH mRNA, the fragment amplified by RT-PCR was used as a probe. These probes were labeled with $\left[\alpha{ }^{32} \mathrm{P}\right]-\mathrm{dCTP}$ using a random primer DNA labeling kit (Boehringer, Mannheim, Germany). Radioactive hybridizing signals on the filters were detected by Bio-Image Analyzer FLA-2000G (Fuji Film, Kanagawa, Japan).

Cloning and sequencing. RT-PCR products were cloned directly into the pT7blue T-vector DNA (Novagen, Madison, WI, USA). The nucleotide sequence was determined on both strands by the dideoxy-chain termination method (18) using Sequenase DNA polymerase (Amersham, Arlington Heights, IL, USA).

\section{RESULTS}

Comparison of HOXD3 mRNA levels, colony morphology, and adhesion to fibronectin in control versus HOXD3 sense and antisense transfected HEL cells

In the previous report (24), we did not examine decreased expression of HOXD3 in HEL cells because we could not obtain HEL clones which express lower levels of HOXD3 mRNA than the control clones. Thus, in the present study, we attempted to carry out repeated experiments in which the empty, HOXD3 sense, and HOXD3 antisense vectors were introduced into HEL cells to obtain novel clones expressing normal, high, and low levels of HOXD3 mRNA.

HEL cells were transfected with either the unmanipulated pMAMneo mammalian expression vector or the vector containing HOXD3 genomic DNA inserted in either the sense or antisense direction. Stable transfectants were selected with G418 and cloned. Cytoplasmic RNA obtained from the HEL transfectants was used for RT-PCR analysis. The RTPCR products were separated on $2 \%$ agarose gels, blotted onto nylon filters, and hybridized with ${ }^{32} \mathrm{P}$-labeled probes. HOXD3 mRNA in the sense transfectant $S 1$ was determined to be 10 -fold higher than in the control cells transfected with the unmanipulated vector (Fig. 1A). The level of HOXD3 expression in the $\mathrm{S} 1$ was similar to those in the E1 and E6 transfectants previously reported (24). The antisense transfectant AS1 did not express any HOXD3
mRNA (Fig. 1A). The blot for $\beta$-actin showed that equal amounts of RNA were used for RT-PCR analysis (Fig. 1A).

The HEL transfectants were seeded at low density in tissue culture flasks, and morphology of colonies that arose from single cells was observed under a phase contrast microscope. All control transfectants examined formed irregular and diffused colonies (Fig. 1B), while the sense transfectant $\mathrm{S} 1$ formed regular and compact colonies that were composed of densely associated cells (Fig. 1B). Colony morphology of the other two transfectants overexpressing HOXD3 was similar to that of S1 (not shown). The antisense transfectant AS1 did not form colonies because almost all cells were floating without forming clusters and failed to attach to the bottom of a flask (Fig. 1B). The antisense transfectant AS3 lacking in HOXD3 expression did not also form colonies (not shown). These results indicate that the formation of colonies in HEL cells was involved in expression of the HOXD3 gene.

To compare the ability to attach to fibronectin among the HEL transfectants, cells were seeded in fibronectin-coated dishes, and the number of attached and unattached cells was counted. The percentage of adhesion in the sense transfectant S1 was 2.4-fold higher than in the control transfectant (Fig. 1C). In contrast, there was a marked decline in the ability to adhere in the antisense transfectant AS1 as compared with that in the control transfectant (Fig. 1C). The above results indicate that the ability to adhere to fibronectin was dependent on levels of HOXD3 expression in HEL cells.

\section{Cadherin gene expression in HOXD3 transfected HEL cells}

Alterations in colony morphology and competence in attaching to fibronectin corresponding to levels of HOXD3 expression prompted us to examine expression of cell-cell and cell-extracellular matrix adhesion molecules in the HEL transfectants. It has been previously reported that the HEL cells overexpressing the HOXD3 gene exhibited an increase in expression of $\beta 3$ integrin mRNA and $\alpha \mathrm{Ilb} \beta 3$ integrin protein (24). In the present study, cadherin expression in the HEL transfectants was investigated by RT-PCR using a primer pair designed within the conserved DNA region encoding C-terminal cadherin proteins. As a result, a 380-bp fragment corresponding to cadherin cDNA was amplified in the antisense transfectant AS1, but not in the control and sense transfectant (Fig. 2A). This cDNA fragment was detected only in the antisense transfec- 


\section{A}

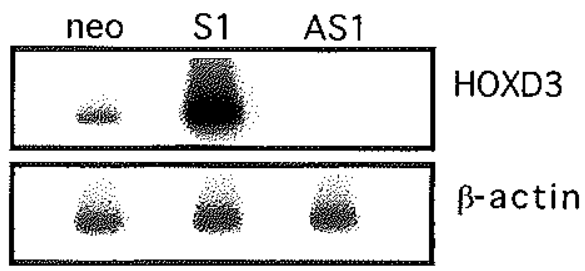

B
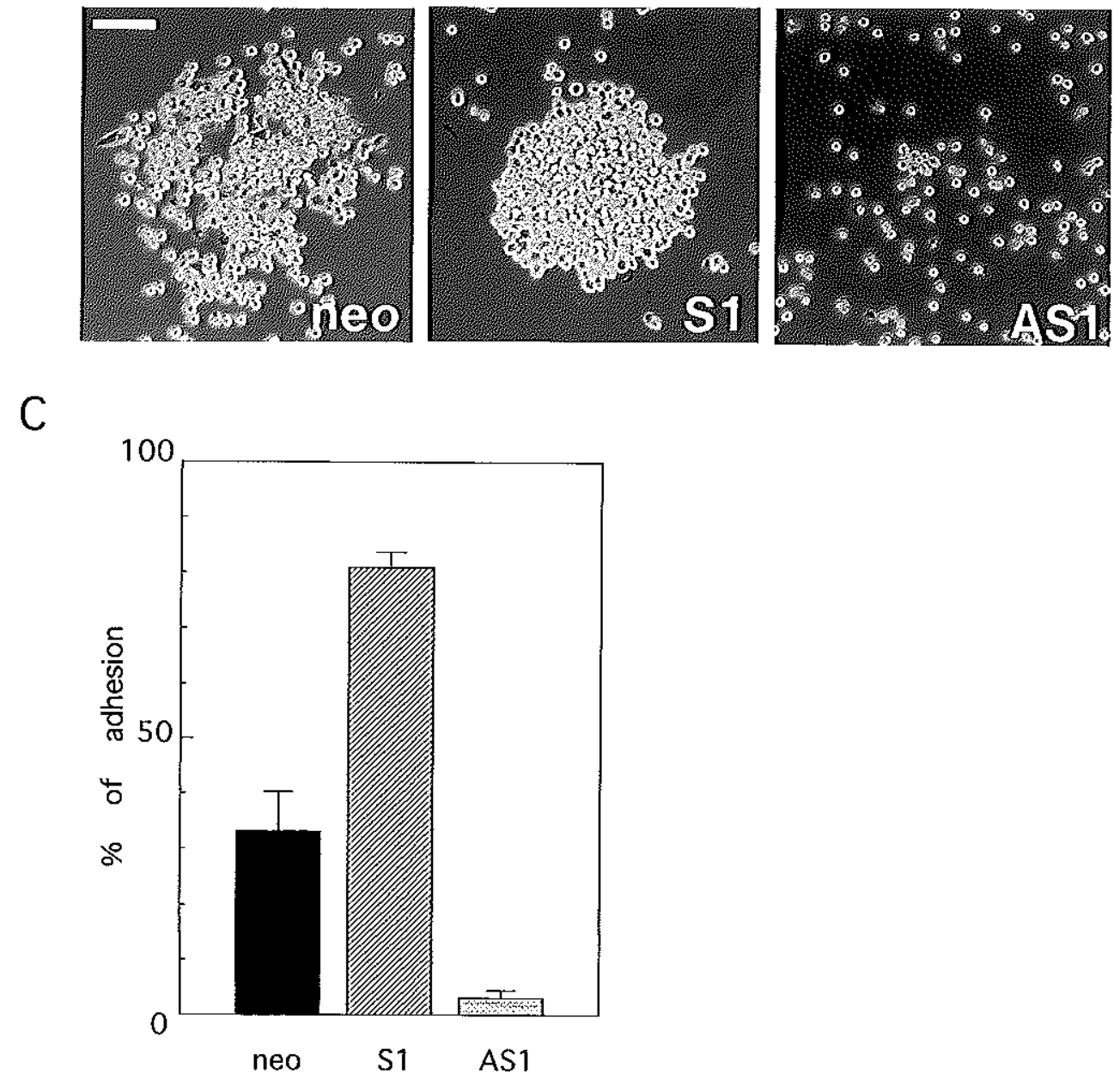

Fig. 1 HOXD3 mRNA levels, colony morphology, and adhesion to fibronectin-coated dishes in control versus HOXD3 sense and antisense transfected HEL cells. A: Cloned HEL cells transfected with the empty vector (neo), pMAMneo-HOXD3 sense (S1) and pMAMneo-HOXD3 antisense (AS1) were seeded at $10^{5}$ cells per $25-\mathrm{cm}^{2}$ tissueculture flasks. After 4-days culture, cytoplasmic RNA was isolated from them, and subjected to RT-PCR at 27 cycles for the HOXD3 cDNA and 17 cycles for the $\beta$-actin cDNA. The RT-PCR products were separated by $2 \%$ agarose gel electrophoresis, blotted onto nylon filters, and hybridized with the ${ }^{32}$ P-labeled HOXD3 or $\beta$-actin probe. B: Phase contrast micrographs of the control (neo), HOXD3 sense (S1), and HOXD3 antisense transfectant (AS1). Cells were plated at $10^{4}$ cells per $25 \mathrm{-cm}^{2}$ tissue-culture flasks. After 7-days culture, micrographs of colonies which arose from a single cell were taken. The AS1 cells were floating without forming colonies. Bar, $100 \mu \mathrm{m}$. C: The control (neo), HOXD3 sense (S1), and HOXD3 antisense transfectant (AS1) were plated at $9 \times 10^{5}$ cells per fibronectin-coated dishes with $3.5 \mathrm{~cm}$ in diameter. After $15-\mathrm{min}$ incubation at $37^{\circ} \mathrm{C}$, nonadherent cells were collected by washing the dishes twice with culture medium and adherent cells were removed by washing PBS containing $0.2 \%$ EDTA. The number of cells in each population was counted, and the percentage of adherent cells per total cells was calculated. Cross bars represent standard deviations of two independent experiments. 
tants expressing lower levels of HOXD3 than the control transfectants (not shown). The cDNA fragment was cloned, and the nucleotide sequence was determined. Consequently, the amino acid sequence deduced from the cDNA nucleotide sequence was found to match exactly to the human cadherin 4 sequence using the BLAST software through the
DDBJ (Fig. 2B and 2C). The mouse orthologue of human cadherin 4 appeared to be R-cadherin because the amino acid sequence of human cadherin 4 showed the highest homology with that of mouse Rcadherin (Fig. 2C). These results suggest that the antisense transfectants, in which HOXD3 expression was suppressed, produced detectable levels of cad-

A

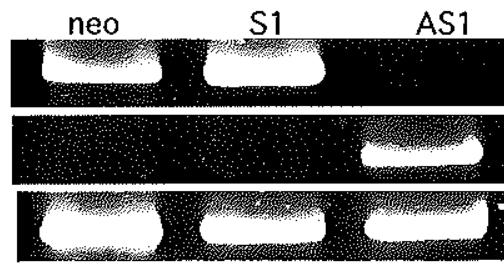

HOXD3

cadherin

$\beta$-actin

B

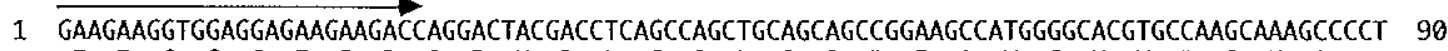

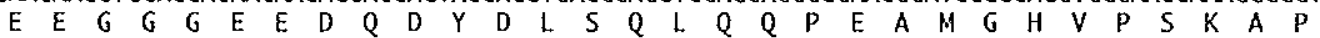

91 GGCGTGCGTCGCGTGGATGAGCGGCCGGTGGGCCCTGAGCCCCAGTACCCGATCAGGCCCATGGTGCCGCACCCAGGCGACATCGGTGAC 180

$\begin{array}{lllllllllllllllllllllllllllllll}G & V & R & R & V & D & E & R & P & V & G & P & E & P & Q & Y & P & I & R & P & M & V & P & H & P & G & D & I & G & D\end{array}$

181 TTCATCAATGAGGGACTCCGCGCTGCTGACAACGACCCCACGGCACCCCCCTATGACTCCCTGCTGGTCTTCGACTACGAGGGGGCGGC 270

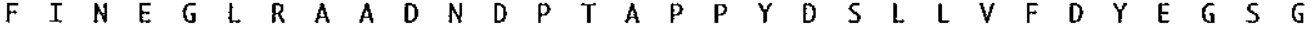

271 TCCACCGCAGGCTCCGTCAGCTCCCTGAACTCATCCAGTTCCGGGGACCAAGACTACGATTACCTCAACGACTGGGGGCCCAGATTCAAG 360 $\begin{array}{llllllllllllllllllllllllllllll}S & T & A & G & S & V & S & S & L & N & S & S & S & S & G & D & Q & D & Y & D & Y & L & N & D & W & G & P & R & F & K\end{array}$

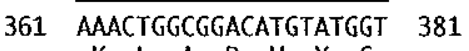

C K L A A D $M$ M Y

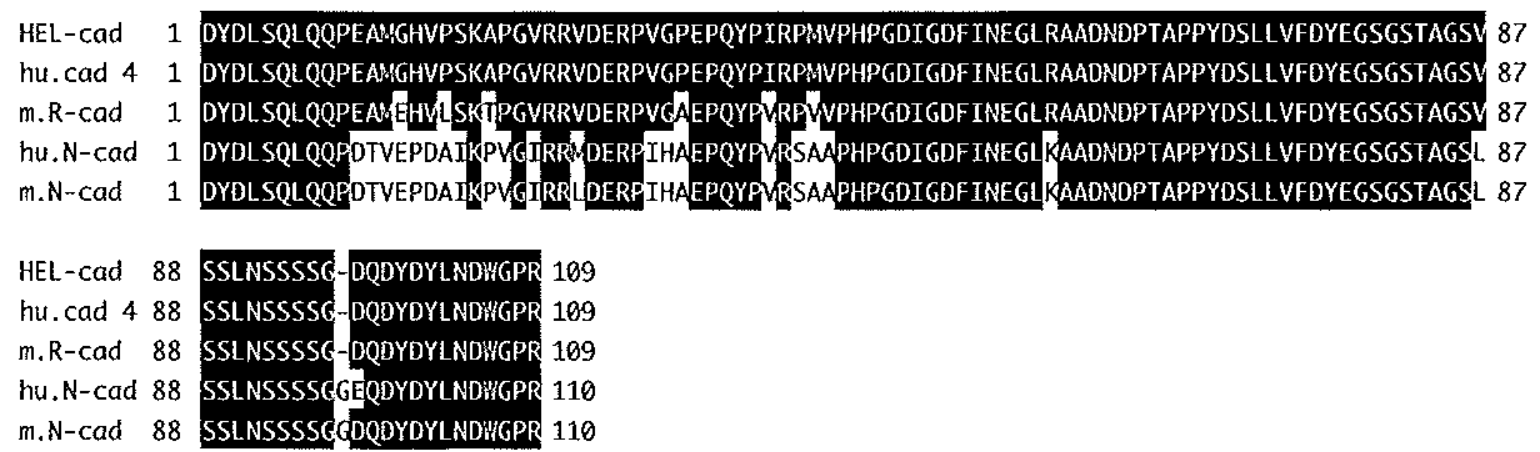

Fig. 2 Cadherin gene expression in HOXD3 transfected HEL cells and identification of the type of cadherin. A: Cytoplasmic RNA was isolated from cloned HEL cells transfected with the empty vector (neo), pMAMneo-HOXD3 sense (S1) and pMAMneo-HOXD3 antisense (AS1), and subjected to RT-PCR at 35 cycles for the HOXD3 cDNA, 45 cycles for the cadherin cDNA, and 22 cycles for the $\beta$-actin cDNA. The annealing temperature for PCR was $65^{\circ} \mathrm{C}$ for the HOXD3 and $\beta$-actin cDNA or $60^{\circ} \mathrm{C}$ for the cadherin cDNA. The RT-PCR products were separated by $7.5 \%$ polyacrylamide gel electrophoresis, and stained by ethidium bromide. B: Nucleotide sequence of the partial cadherin cDNA and amino acid sequence deduced from the cDNA nucleotide sequence. The cadherin cDNA fragment was cloned into the pT7blue T-vector DNA, and the nucleotide sequence of the cDNA was determined. The arrows indicate positions of the primers used to detect cadherin cDNA. C: Comparison between the amino acid sequences of a partial protein encoded by the amplified cadherin cDNA fragment and other known cadherin proteins. HEL-cad represents the cadherin protein encoded by the cadherin cDNA fragment amplified in HOXD3 antisense transfected HEL cells. Other known cadherins: hu. cad 4, human cadherin 4; m. R-cad, mouse R-cadherin; hu. N-cad, human N-cadherin; $\mathrm{m}$. N-cad, mouse N-cadherin. Boxes shaded in black show identical amino acids. Dashes indicate gaps introduced to maximize sequence similarity. 
herin 4 mRNA.

Cadherin and integrin gene expression in control versus HOXD3 sense and antisense transfected HEL cells

Cytoplasmic RNA was isolated from the transfectants. This RNA was subjected to RT-PCR analysis for detection of HOXD3 and cadherin 4 expression, and subjected to Northern analysis for detection of $\beta 1$ and $\beta 3$ integrin expression (Fig. 3). By PCR at 30 cycles, the cadherin 4 fragments were detected in the antisense transfectants AS1 and AS2 expressing lower levels of HOXD3 than the control transfectant, but not detected in the control and sense transfectants. By PCR at 35 cycles, a very faint signal corresponding to cadherin $4 \mathrm{mRNA}$ appeared in the control transfectant, but did not appear in the sense transfectants. These results indicate that HOXD3 had an inhibitory effect on cadherin 4 gene expression in HEL cells. In contrast, the sense transfectants S1 and S2 overexpressing the HOXD3 gene expressed increased levels of $\beta 3$ integrin mRNA and the antisense transfectant AS1 which did not express the HOXD3 gene at all expressed a reduced level of $\beta 3$ integrin mRNA. This shows that HOXD3 promoted the expression of $\beta 3$ integrin mRNA in HEL cells, as we confirmed in the previous report (24). $\beta 1$ integrin mRNA levels were similar among the transfectants, suggesting that HOXD3 had no effect of $\beta 1$ integrin gene expression in HEL cells. Hybridization of the blot with $\beta$-actin and G3PDH probes served as positive controls of RTPCR and Northern hybridization, respectively.

\section{DISCUSSION}

In the present study, we have revealed that varied levels of HOXD3 expression in HEL cells resulted in differences in colony morphology and expression of cell adhesion molecules. The HEL cells deficient in HOXD3 gene expression failed to form colonies on tissue-culture surface and had a low affinity for fibronectin matrix, expressing a very low level of $\beta 3$ integrin mRNA. Although the cells floated without cell-cell contact in tissue-culture flasks, they exhibited cadherin 4 gene expression. In proportion to increasing levels of HOXD3 expression, HEL cells acquired the ability to form colonies and a higher affinity for fibronectin. The HEL cells overexpressing HOXD3 formed compact colonies composed of condensed cells on tissue-culture surface and attached efficiently to fibronectin. In the cells overexpressing HOXD3, cadherin 4 gene expression was replaced by $\beta 3$ integrin gene expression. These findings demonstrate that the ability to form colonies and attach to fibronectin in HEL cells was dependent on levels of HOXD3 expression, and that HOXD3 served as the switch from cadherin 4 to $\beta 3$ integrin gene expression. This study is the first to report a switching mechanism involving HOXD3, though HOXD3 has been previously reported to stimulate $\beta 3$ integrin

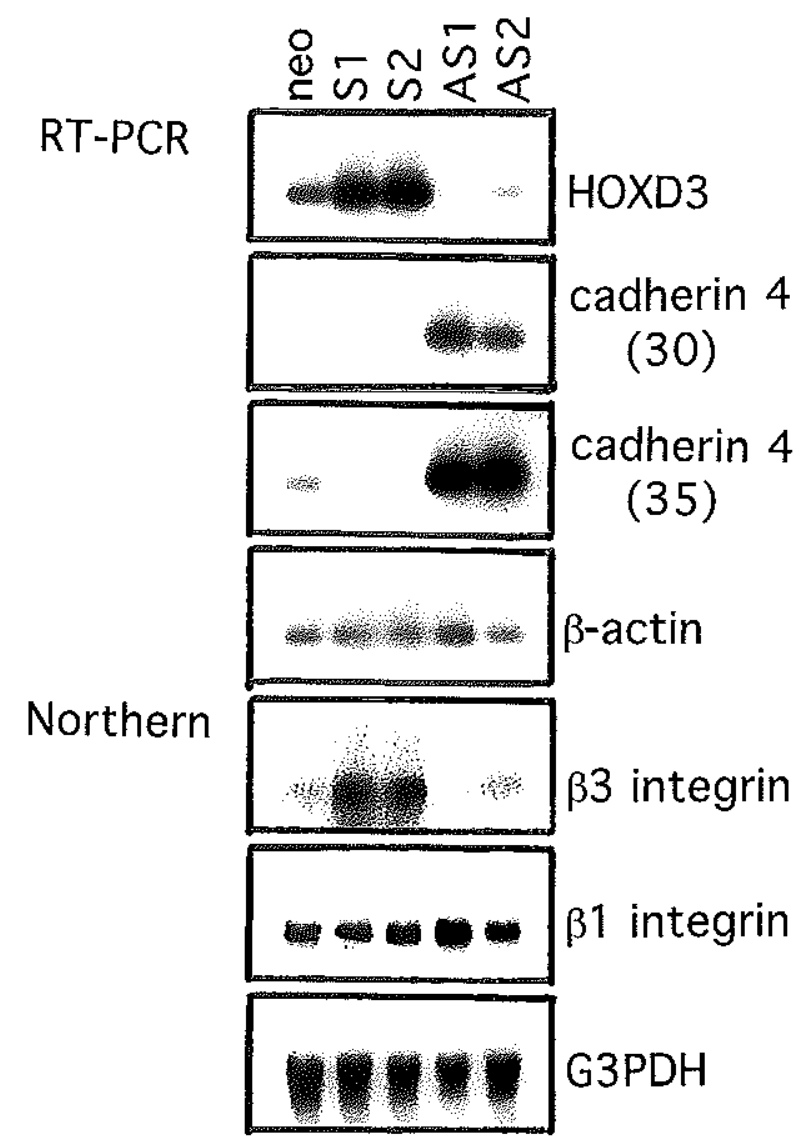

Fig. 3 Cadherin and integrin gene expression in control versus HOXD3 sense and antisense transfected HEL cells. Cytoplasmic RNA was isolated from cloned HEL cells transfected with the empty vector (neo), pMAMneo-HOXD3 sense (S1 and S2) and pMAMneoHOXD3 antisense (AS1 and AS2), and subjected to RTPCR and Northem hybridization. For RT-PCR analysis, PCRs were run at 30 cycles for the HOXD3 cDNA, 30 or 35 cycles for the cadherin 4 cDNA, and 17 cycles for the $\beta$-actin cDNA. The RT-PCR products were separated by $2 \%$ agarose gel electrophoresis, blotted onto nylon filters, and hybridized with the HOXD3, mouse Rcadherin cDNA, or $\beta$-actin probe. For Northern analysis, a total of $20 \mu \mathrm{g}$ cytoplasmic RNA was electrophoresed on $1 \%$ agarose-formaldehyde gels, blotted onto nylon filters, and hybridized with the $\beta 1$ integrin, $\beta 3$ integrin, or G3PDH cDNA probe. 
gene expression in several types of cells $(2,9,24)$.

Our results demonstrate that, in HEL cells, a decline of HOXD3 expression gave rise to the conversion of $\beta 3$ integrin which directs cell-extracellular matrix interaction into cadherin 4 which mediates cell-cell adhesion. However, it is curious that the HEL cells expressing cadherin 4 were free-floating. As for the reason why the cadherin-expressing cells could not form aggregates, we presume the following two possibilities. First, the cells might not have a sufficient amount of cadherin 4 protein on their surfaces to form cell-cell adhesion. Actually, cadherin $4 \mathrm{mRNA}$ detected by RT-PCR analysis in the cells could not be found by Northern analysis using total RNA extracted from the cells. Second, cadherin 4 protein expressed on the surfaces of the cells was likely to fail to function because the cells did not possess a complete intracellular system necessary for cell-cell attachment mediated by cadherin molecules.

The bone marrow provides a hematopoietic microenvironment for proliferation and differentiation of hematopoietic progenitor cells (6). Various adhesion molecules including the integrin family contribute to the development of the progenitor cells through cellextracellular matrix interaction (11). On the other hand, E-cadherin, which mediates cell-cell communications, has been reported to play an important role in the maturation of erythrocytes within the bone marrow (1). Therefore, judging from our findings, it is very likely that, in developing hematopoietic cells, the switchover of cell-cell to cell-extracellular matrix interaction takes place under the direct or indirect control of transcription factors such as homeodomain protein HOXD3.

Mice lacking the function of Hoxd-3, a counterpart of human HOXD3, display homeotic transformations of the anterior arch of the atlas into an extension of the basioccipital bone of the skull, and the axis into an atlas-like structure (4). From these observations, Hoxd-3 is assumed to control the proliferation rates of the mesenchymal condensation that give rise to the vertebral cartilages (4). The mesenchymal condensation in skeletal pattern formation has been reported to correlate with modulation of cell-extracellular matrix interaction $(7,8)$. Thus, although our findings are still restricted to several cell lines, the changes in colony morphology and avidity for fibronectin, dependent on levels of HOXD3 expression, may be closely related to the cellular dynamics in skeletal development in vertebrates. We anticipate that the switching from cadherin to integrin gene expression by HOXD3 may be involved in the epithelial-to-mesenchymal transformation of cells during carcinogenesis and embryonic development.

\section{Acknowledgements}

We thank Dr. Masatoshi Takeichi for the gift of the mouse R-cadherin cDNA. This work was supported in part by a Grant-in-Aid for Scientific Research (C) from the Ministry of Education, Science, Sports and Culture of Japan and by Tokai University School of Medicine Research Aid.

\section{REFERENCES}

1. Armeanu, S., Buhring, H. J., Reuss-Borst, M., Muller, C. A. and Klein, G. (1995) E-cadherin is functionally involved in the maturation of the erythroid lineage. J. Cell Biol. 131, 243249.

2. Boudreau, N., Andrews, C., Srebrow, A., Ravanpay, A. and Cheresh D, A. (1997) Induction of the angiogenic phenotype by Hox D3.J. Cell Biol. 139, 257-264.

3. Brown, IV. M., Zhou, L. and Taylor, G. R. (1994) The nucleotide sequence of the murine Hox-D3 (Hox-4.1) gene reveals extensive identity with the human protein. Biochim. Biophys. Acta. 1219, 219-222.

4. Condie, B. G. and Capecchi, M. R. (1993) Mice homozygous for a targeted disruption of Hoxd-3 (Hox-4.1) exhibit anterior transformations of the first and second cervical vertebrae, the atlas and the axis. Development 119, 579-595.

5. Condie, B. G. and Capecchi, M. R. (1994) Mice with targeted disruptions in the paralogous genes hoxa-3 and hoxd-3 reveal synergistic interactions. Nature 370, 304-307.

6. Dexter, T. M., Coutinho, L. H., Spooncer, E., Heyworth, C. M., Daniel, C. P., Schiro, R., Chang, J. and Allen, T. D. (1990) Stromal cells in haemopoiesis. Ciba Found. Symp. $148,76^{-86}$.

7. Downie, S. A. and Newman, S. A. (1995) Different roles for fibronectin in the generation of fore and hind limb precartilage condensations. Dev. Biol. 172, 519-530.

8. Hall, B. K. and Miyake, T. (2000) All for one and one for all: condensations and the initiation of skeletal development. Bioessay's 22, 138-147.

9. Hamada, J., Omatsu, T., Okada, F., Furuuchi, K., Okubo, Y., Takahashi, Y., Tada, M., Miyazaki, Y., Taniguchi, Y., Shirato, H., Miyasaka, K. and Moriuchi, T. (2001) Overexpression of homeobox gene HOXD3 induces coordinate expression of metastasis-related genes in human lung cancer cells. Imt. $J$. Cancer 93, 516-525.

10. Kappen, C., Schughart, K. and Ruddle, F. H. (1993) Early evolutionary origin of major homeodomain sequence classes. Genomics 18, 54-70.

11. Liesveld, J. L., Winslow, J. M., Frediani, K. E., Ryan, D. H. and Abboud, C. N. (1993) Expression of integrins and examination of their adhesive function in normal and leukemic hematopoietic cells. Blood 81, 112-121.

12. Manley, N. R. and Capecchi, M. R. (1997) Hox group 3 paralogous genes act synergistically in the fonmation of somitic and neural crest-derived structures. Dev. Biol. 192, 274-288.

13. Manley, N. R. and Capecchi, M. R. (1998) Hox group 3 paralogs regulate the development and migration of the thymus, 
thyroid, and parathyroid glands. Dev. Biol. 195, 1-15.

14. Matsunami, H., Miyatani, S., Inoue, T., Copeland, N. G., Gilbert, D. J., Jenkins, N. A. and Takeichi, M. (1993) Cell binding specificity of mouse R-cadherin and chromosomal mapping of the gene. J. Cell Sci. 106, 401-409.

15. McGinnis, W. and Krumlauf, R. (1992) Homeobox genes and axial patterning. Cell $68,283-302$.

16. Rijli, F. M., Mark, M., Lakkaraju, S., Dierich, A., Dolle, P. and Chambon, P. (1993) A homeotic transformation is generated in the rostral branchial region of the head by disruption of Hoxa-2, which acts as a selector gene. Cell 75, 1333-1349.

17. Sambrook, J., Fritsch, E. F. and Maniatis, T. (1989) Molecular Cloning: A Laboratory Manual (ed 2). Cold Spring Harbor Laboratory.

18. Sanger, F., Nicklen, S. and Coulson, A. R. (1977) DNA sequencing with chain-terminating inhibitors. Proc. Natl. Acad. Sci. USA 74, 5463-5467.

19. Scott, M. P. (1992) Vertebrate homeobox gene nomenclature. Cell 71, 551-553.

20. Searcy, R. D. and Yutzey, K. E. (1998) Analysis of Hox gene expression during early avian heart development. Dev $D y n$.
213, 82-91.

21. Tan, D. P, Shao, X., Pu, L., Guo, V. and Nirenberg, M. (1996) Sequence and expression of the murine Hoxd-3 homeobox gene. Proc. Natl. Acad. Sci. USA 93, 8247-8252.

22. Taniguchi, Y., Inoko, H., Ando, A., Iwasaki, K., Fujii, A., Suemizu, H., Yoshimura, S. and Moriuchi, T. (1991) Structural analysis of the human HOX4A homeobox gene. Nucleic Acids Symp. Ser: 25, 31-32.

23. Taniguchi, Y., Fujii, A. and Moriuchi, T. (1992) Cloning and sequencing of the human homeobox gene HOX4A. Biochim. Biophys. Acta 1132, 332-334.

24. Taniguchi, Y., Komatsu, N. and Moriuchi, T. (1995) Overexpression of the HOX4A (HOXD3) homeobox gene in human erythroleukemia HEL cells results in altered adhesive properties. Blood 85, 2786 $\cdots 2794$.

25. Taniguchi, Y., Matsuzaka, Y., Fujimoto, H., Miyado, K., Kohda, A., Okumura, K., Kimura, M. and Inoko H. (1998) Nucleotide sequence of the Ring 3 gene in the class II region of the mouse $\mathrm{MHC}$ and its abundant expression in testicular germ cells. Genomics 51, 114-123. 